\title{
A comparison of rheumatoid arthritis in Australia and China
}

\author{
HELEN MORAN, ${ }^{1}$ SHUN-LE CHEN ${ }^{2}$ K D MUIRDEN, ${ }^{1}$ SHAO-JI JIANG, \\ YUE-YING GU, ${ }^{2}$ JOHN HOPPER, ${ }^{2}$ PEI-LIN JIANG ${ }^{2}$ GARY LAWLER, \\ AND REN-BIAO CHEN ${ }^{2}$
}

From the ${ }^{1}$ Rheumatology Unit, Royal Melbourne Hospital, Australia; and the ${ }^{2}$ Rheumatology Unit, Shanghai Third People's Hospital, Shanghai, China

SUMMARY A comparison was made of two series of consecutive outpatients with a presumptive diagnosis of rheumatoid arthritis (RA) attending referral centres in Melbourne and Shanghai. No significant differences were observed in disease onset, course, presence of antinuclear antibodies (ANA), or seropositivity. In the Australian series there was a higher frequency of nodules, Raynaud's phenomenon, carpal tunnel syndrome, and 'classical' in comparison with 'definite' disease, and a lower frequency of lymphadenopathy and hepatomegaly. Joint tenderness and soft tissue swelling tended to be more marked in the Chinese series, while deformity and limited range of movement were less severe. Drug therapy was similar overall but influenced by drug availability. Peptic ulceration was recorded in $28 \%$ of the Australian series but in only $6 \%$ of the Chinese; although $25 \%$ of the Chinese were receiving antacids and $6 \%$ antiulcerants. $x$ Rays of hands and feet showed more severe disease in the Australian series. The older age group and longer duration of the disease in the Australian patients, who had more chronic and less active disease, may have influenced some of these results.

A good deal of mystery surrounds the prevalence and severity of rheumatoid arthritis (RA) in the Asian and Western Pacific region. The identification of any regional differences in disease distribution or clinical features could provide important leads to aetiology. Although diagnostic criteria used in different countries have varied, prevalence determinations have shown less variability than for most diseases. A 15 year longitudinal population study of RA in Japan, ' however, indicated that both incidence and prevalence (the latter being $0.3 \%$ ) were significantly lower than in Western Europe and the USA, where the prevalence is between 1 and $3 \% .^{23}$ Such evidence as is available indicates that the European populations in Australia and New Zealand have figures similar to those of Europe. New Zealand studies suggest that Polynesians have lower rates of RA than European New Zealanders, ${ }^{4}$ and there is some largely anecdotal evidence that in other Asian populations the disease is milder and nodules are rare. A survey of a Chinese population

Accepted for publication 20 January 1986.

Correspondence to Dr Helen Moran, Rheumatology Unit, Royal Melbourne Hospital, Victoria 3050, Australia. living on a rural island off the coast of China showed a prevalence of RA of no more than $0 \cdot 3 \% .{ }^{5}$ It was? stated that no clinically unusual features were recognised in these cases, but only 17 subjects with definite RA were examined. No prevalence study has so far been completed in China. It has been suggested, however, that RA as a whole seems 0 milder in severity in China than in western countries. ${ }^{6}$ Chang Nai-Cheng in Beijing reports that ${ }^{\circ}$ according to radiological staging of disease pro- $\rightarrow$ gression and classification of joint function the very severe stage 4 and class IV patients were not $N$ commonly seen in his clinic, the incidence of rheumatoid nodules was relatively low $(5-13 \%)$, N and pulmonary involvement was rarely seen. ${ }^{6}$ The $\omega$ hypothesis to be considered in this study is that RAP in China is a less severe disease with fewer visceralo manifestations than in a European population in $\Phi$ Australia. A comparison was therefore made of $\stackrel{\mathcal{P}}{?}$ consecutive outpatients attending a tertiary referral $\underline{T}$ centre in Melbourne and Shanghai having classic or $\frac{\mathrm{O}}{\mathbb{D}}$

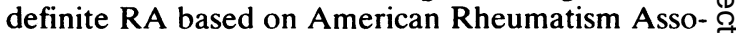
ciation (ARA) criteria. The protocol considers $\stackrel{\Phi}{\mathscr{D}}$ onset and course of disease, the distribution of involved joints, severity of synovitis, extent of 
deformities, and extra-articular manifestations. $x$ Rays of hands, feet, and chest were compared and HLA including DR typing undertaken. Consistency in recording clinical data was based on the fact that Dr Chen has recently spent a year of postgraduate clinical work at the Melbourne Unit.

\section{Methods}

The study was carried out at a rheumatology outpatients clinic in a major teaching hospital in Melbourne and Shanghai-both established tertiary referral centres in their cities. Comparison was made of 100 consecutive outpatients with a presumptive diagnosis of RA attending the two clinics. Both new referrals and follow up cases were included. In the Australian series only 82 patients were examined over subsequent weeks after the consecutive 100 cases had been registered. Of the 18 patients not included in the study, the diagnosis was found to be incorrect in three and three died before being adequately assessed. In the other 12 full follow up was not possible as the cases had moved either interstate or to other hospital units. The initial assessment of these 12 cases did not show that they differed in overall severity from the remainder of the series.

The diagnosis of RA was made according to standard ARA criteria and classified as classical or definite disease. No probable cases were included. The disease onset was defined as insidious if evolution occurred gradually over weeks or months, and acute if symptoms developed within days. Patients were asked to describe the course of their disease as towards remission, or as intermittent, fluctuating, sustained, or progressive. Such inquiries were aided by the use of the diagram (Fig. 1). Apart

(1)

Remission

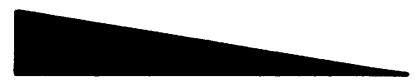

(2)

Intermittent

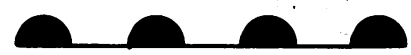

(3)

Fluctuating

Sustained

Progressive

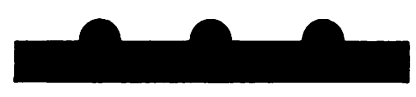

\section{(6) Other}

Fig. 1 Course of rheumatoid arthritis. from age, sex, and disease duration the patient's occupation and a past history of tuberculosis or rheumatic fever were recorded. The presence of associated medical conditions including peptic ulcer, diabetes mellitus, ischaemic heart disease, etc. were noted. Past and present symptoms and signs suggestive of extra-articular involvement were sought and these included for example, Raynaud's phenomena, peripheral neuropathy, mononeuritis, cardiac and pulmonary involvement, and symptoms related to the eyes. Schirmer's test for conjuctivitis sicca was performed and the grip strength recorded. A general physical examination and a detailed musculoskeletal examination were performed. Particular emphasis was placed on distribution of involved joints and tendons, the severity of synovitis, and the extent of deformities. Details of past and present antirheumatic drug therapy were noted, as was the use of non-rheumatic drugs. Inquiries were made as to the use of herbalist, folk, or traditional drugs. Surgical procedures were also recorded.

$x$ Rays included in the study were one view of both hands, including wrists, one view of the feet, and a posteroanterior chest $x$ ray. The hands and feet $x$ rays were ranked as follows: $1=$ normal; $2=$ earlysoft tissue swelling and periarticular osteoporosis; $3=$ mild-soft tissue swelling, osteoporosis, and a definite erosion or two; 4 and $5=$ moderate $A$ and $B$ - these showed severe erosions, joint space narrowing, and some deformity, with $A$ being less severe than $B ; 6=$ severe-such cases showed gross erosions, subluxation, deformity, joint space narrowing, and occasionally ankylosis. Films were initially graded independently by two rheumatologists and a radiologist in each country. In each unit where a discrepancy was found the films were reevaluated by the panel of three observers. Films were exchanged between the two centres and results compared. Of $24 x$ rays of the hands exchanged, 19 were consistent in the two centres and five varied by only one grade. The chest $x$ rays were classified as (1) normal, (2) mild increase in lung markings, (3) moderate increase in lung markings, (4) interstitial fibrosis, (5) pleural adhesions, (6) old (chronic) tuberculosis, (7) an enlarged heart.

Photographs of the patient's hands and feet were taken to assist in maintaining consistency in recording joint deformity. Haematological, biochemical, and serological tests were performed and these included a haemoglobin, white cell count and differential count, platelet count, erythrocyte sedimentation rate (ESR) measurement performed by the Westergren method, serum creatinine determination, and urinalysis. Rheumatoid factor was measured by both the latex and sheep cell agglutination tests, and the kits used for the Chinese patients 
were supplied by the Melbourne unit. Antinuclear antibody titres and pattern were based on $\mathrm{HEp}_{2}$ cells, also supplied by the Melbourne unit. HLA including DR typing was also used, and HLA typing trays and sera were supplied to Shanghai by the Royal Melbourne Hospital. The HLA data will not be included in this report as further population cross checking is necessary. Data were coded and entered on the University of Melbourne VAX computer system using the database system DATATRIEVE. Statistical analyses (contingency tables, $t$ tests) were carried out using the statistical package for the social sciences. Interpretation of significance statements must be tempered by multiple comparison considerations.

\section{Results}

As expected the majority of cases were female, $76 \%$ of the 100 Chinese and $69 \%$ of the 82 Australians, with the difference being not significant. The Australian patients were significantly older, with a mean age of 60 years (SD 14) compared with 45 years (SD 13) in China $(p<0 \cdot 001)$. Disease duration was considerably longer in Australia 12.6 years (SD 9) compared with China 6.3 years (SD 6) $(\mathrm{p}<0 \cdot 001)$. A past history of tuberculosis was present in $17 \%$ of Chinese patients but was absent from the Australian series $(p<0.0002)$ (Table 1). A past history of rheumatic fever occurred in $3 \%$ of the Chinese and $6 \%$ of the Australian patients, the difference being not significant. The presence of associated medical conditions-diabetes mellitus, hypertension, ischaemic heart disease, chronic bronchitis and emphysema, and peptic ulceration are shown in Table 1 , as is a family history of inflammatory arthritis. A weak significant difference was shown for ischaemic heart disease, possibly reflecting the

Table 1 Features from history (figures in percentages)

\begin{tabular}{|c|c|c|c|}
\hline & China & Australia & Significance \\
\hline $\begin{array}{l}\text { Family history of } \\
\text { inflammatory arthritis }\end{array}$ & 7 & 18 & NS \\
\hline Past history of tuberculosis & 17 & 0 & 0.0002 \\
\hline $\begin{array}{l}\text { Past history of rheumatic } \\
\text { fever }\end{array}$ & 3 & 6 & NS \\
\hline $\begin{array}{l}\text { Associated diabeties } \\
\text { mellitus }\end{array}$ & 1 & 4 & NS \\
\hline Associated hypertension & 19 & 27 & NS \\
\hline $\begin{array}{l}\text { Associated ischaemic } \\
\text { heart disease }\end{array}$ & 2 & 10 & $0 \cdot 05$ \\
\hline $\begin{array}{l}\text { Associated chronic } \\
\text { bronchitis and } \\
\text { emphysema }\end{array}$ & 11 & 15 & NS \\
\hline Associated peptic ulcer & 6 & 28 & 0.0001 \\
\hline
\end{tabular}

older population, and a strong difference was shown $\frac{\mathrm{D}}{\mathrm{C}}$ for peptic ulceration.

As indicated, all cases fulfilled criteria for either $\overrightarrow{\overrightarrow{\vec{S}}}$ classical or definite disease but there was a significant difference in that $82 \%$ of the Australian series compared with $50 \%$ of the Chinese series showed

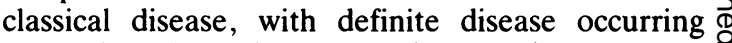
respectively in $18 \%$ and $50 \%(\mathrm{p}<0.001)$. There was no significant difference in disease onset, with $31 \%$ s of Australian and $37 \%$ of Chinese having an acute $\vec{\circ}$ onset, while the figures for an insidious onset were $\overrightarrow{\vec{\omega}}$ respectively $69 \%$ and $63 \%$ (NS). The disease course ${ }^{\omega}$ also showed no significant differences, with virtually equal numbers in China and Australia being included in each grouping (Table 2). Specific symp- of toms listed in the protocol included fever, of Raynaud's phenomenon, carpal tunnel syndrome, mononeuritis, dry eyes, episcleritis, psoriasis or $\circ$ other rashes, pericarditis, myocarditis, or pleurisy. Raynaud's phenomenon was more common in the Australian series $(\mathrm{p}<0.0002)$, while a past history of $\stackrel{\bigodot}{\gtrless}$ carpal tunnel syndrome occurred in $22 \%$ of the $\vec{\bullet}$ Australian series and only $2 \%$ of the Chinese 8 $(p<0 \cdot 001)$. Symptoms (but not signs) of a peripheral neuritis were commoner in the Chinese series, where they represented $17 \%$ compared with $2 \%$ of the Australian series $(p<0.005)$. When it came to physical signs, subcutaneous and tendon nodules were much commoner in the Australian series, while lymphadenopathy, hepatomegaly, but not splenomegaly were commoner in China (Table 3 ). Ocular signs were uncommon in both series, and the same was true of pulmonary, cardiac, and neurological signs. Nail fold infarcts were also uncommon, and leg ulcers were more frequent in the Australian series (see Table 3). A positive Schirmer's test for lacrymal secretion (10 mm or less) was found in $64 \%$ of Chinese and $40 \%$ of Australians $(p<0 \cdot 05)$.

EXAMINATION OF THE JOINTS

Examination of the joints showed a number of $\frac{D}{O}$ striking differences. Ulnar drift of the fingers was measured as,+++ , or +++ . Deformity was more $\tilde{N}$ frequent in the Australian series, and the severer

Table 2 Disease course (figures in percentages)

\begin{tabular}{lccc}
\hline & China & Australia \\
\hline Remission & 6 & 12 \\
Intermittent & 16 & 10 \\
Fluctuating & 48 & 46 & 7 \\
Sustained & 6 & 21 & 4 \\
Progressive & 24 & 0 & \\
Other & 0 & & \\
\hline
\end{tabular}

The differences are not significant. 
Table 3 Present clinical features (figures in percentages)

\begin{tabular}{lcll}
\hline & China & Australia & Significance \\
\hline Raynaud's phenomenon & 4 & 17 & $0 \cdot 0002$ \\
Subcutaneous nodules & 0 & 20 & $0 \cdot 0001$ \\
Tendon nodules & 2 & 10 & $0 \cdot 01$ \\
Lymphadenopathy & 30 & 3 & $0 \cdot 0001$ \\
Splenomegaly & 4 & 2 & NS \\
Hepatomegaly & 12 & 1 & $0 \cdot 01$ \\
Peripheral neuritis & & & \\
$\quad$ (on examination) & 2 & 1 & NS \\
Nail fold infarcts & 1 & 3 & NS \\
Leg ulcers & 1 & 4 & $0 \cdot 05$ \\
Episcleritis & 0 & 1 & NS \\
\hline
\end{tabular}

Table 4 Ulnar drift (figures in percentages)

\begin{tabular}{lllcl}
\hline & Overall & + & ++ & +++ \\
\hline China & 23 & L 11 & 5 & 0 \\
Australia & 43 & R 15 & 7 & 0 \\
& & L 15 & 13 & 9 \\
Significance & 0.0001 & & & \\
\hline
\end{tabular}

deformities were also marked in this group (Table 4). The Australians also showed a marked reduction in grip strength compared with that of the Chinese $(p<0.0001)$. The peripheral joints were assessed with regard to tenderness, soft tissue swelling, bony swelling, deformity, and reduction in range of motion. These features were graded as mild, moderate, or severe. The results are indicated in Table 5, and marked differences in the two series are to be noted. Overall there was a trend for joint tenderness and soft tissue swelling to be more marked in the Chinese series, while deformity and limited range of motion were more a feature in the Australian group. Cervical spine limitation of movement was equal in the two series, but tenderness was more marked in China. The acromioclavicular, temporomandibular, and ankle joints were more commonly involved in China, while the subtalar joint was recorded as being more affected in Australia. Some differences in examination technique may explain the latter contrast.

T RE A T M E N T

Virtually all patients from both series had been

Table 5 Joint examination (figures in percentages)

\begin{tabular}{|c|c|c|c|c|c|}
\hline & & Tenderness & $\begin{array}{l}\text { Soft tissue } \\
\text { swelling }\end{array}$ & Deformity & Range of motion \\
\hline \multirow[t]{2}{*}{ Proximal interphalangeal (PIP) } & China & $77^{*}$ & $70^{*}$ & 0 & 1 \\
\hline & Aust & 28 & 30 & 21 & $13^{*}$ \\
\hline \multirow[t]{2}{*}{ Metacarpophalangeal (MCP) } & China & $71^{*}$ & 60 & 23 & 9 \\
\hline & Aust & 37 & 62 & 43 & 13 \\
\hline \multirow[t]{2}{*}{ Wrist } & China & $63^{*}$ & 58 & 3 & 65 \\
\hline & Aust & 34 & 41 & 9 & 67 \\
\hline \multirow[t]{2}{*}{ Elbow } & China & $54^{*}$ & 19 & 10 & 32 \\
\hline & Aust & 11 & 10 & $44^{*}$ & 41 \\
\hline \multirow[t]{2}{*}{ Shoulder } & China & $42^{*}$ & 2 & 0 & 7 \\
\hline & Aust & 4 & 1 & 1 & $46^{*}$ \\
\hline \multirow[t]{2}{*}{ Acromioclavicular } & China & 20 & 1 & 0 & 1 \\
\hline & Aust & 3 & 1 & 0 & 0 \\
\hline \multirow[t]{2}{*}{ Sternoclavicular } & China & 4 & 0 & 0 & 1 \\
\hline & Aust & 3 & 1 & 0 & 0 \\
\hline \multirow[t]{2}{*}{ Temporomandibular } & China & 14 & 1 & 0 & 4 \\
\hline & Aust & 5 & 1 & 0 & 1 \\
\hline \multirow[t]{2}{*}{ Cervical spine } & China & $25^{*}$ & 0 & 0 & 31 \\
\hline & Aust & 2 & 0 & 0 & 33 \\
\hline \multirow[t]{2}{*}{ L-S spine } & China & 8 & 0 & 0 & 5 \\
\hline & Aust & 6 & 0 & 0 & 11 \\
\hline \multirow[t]{2}{*}{ Hip } & China & $16^{*}$ & 1 & 0 & 5 \\
\hline & Aust & 0 & 2 & 1 & 15 \\
\hline \multirow[t]{2}{*}{ Knee } & China & $44^{*}$ & 26 & 12 & 30 \\
\hline & Aust & 11 & 30 & 19 & 30 \\
\hline \multirow[t]{2}{*}{ Ankle } & China & $42 *$ & $26^{*}$ & 0 & $37^{*}$ \\
\hline & Aust & 6 & 6 & 0 & 14 \\
\hline Subtalar & China & $\mathrm{NR}+$ & NR & NR & 2 \\
\hline \multirow{2}{*}{ Metatarsophalangeal (MTP) } & $\begin{array}{l}\text { Aust } \\
\text { China }\end{array}$ & $35^{*}$ & 22 & 1 & $\begin{array}{c}44^{*} \\
0\end{array}$ \\
\hline & Aust & 13 & 18 & 0 & 0 \\
\hline
\end{tabular}

${ }^{*}$ Difference significant at $0 \cdot 05$ level.

$\dagger \mathrm{NR}=$ not recorded. 
treated with non-steroidal anti-inflammatory drugs (NSAIDs) at some stage in the disease course. Current therapy with NSAIDs is indicated in Table 6 , but some account must be taken of the availability of drugs in the two countries. At the time the study was conducted piroxicam was not available in Australia and a large proportion of the category called 'other' consisted of sulindac. Availability of drugs explains some of the major differences in the use of the slow acting antirheumatic drugs (SAARDs; Table 7). Gold was not available in

Table 6 Current therapy with non-steroidal anti-inflammatory drugs (figures in percentages)

\begin{tabular}{lcc}
\hline & China & Australia \\
\hline Salicylate & 3 & 37 \\
Indomethacin & 44 & 27 \\
Phenylbutazone & 1 & 0 \\
Ibuprofen & 56 & 9 \\
Naproxen & 4 & 26 \\
Other & 22 & 51 \\
Total receiving NSAIDs & 85 & 73 \\
\hline
\end{tabular}

Table 7 Therapy with slow acting antirheumatic drugs (figures in percentages)

\begin{tabular}{|c|c|c|c|c|}
\hline & \multicolumn{2}{|c|}{ Current } & \multicolumn{2}{|l|}{ Past } \\
\hline & China & Australia & China & Australia \\
\hline Gold & 0 & 9 & 0 & 59 \\
\hline D-Penicillamine & 21 & 15 & 36 & 21 \\
\hline Antimalarial & 3 & 9 & 2 & 12 \\
\hline Azathioprine & 0 & 4 & 0 & 2 \\
\hline Cyclophosphamide & 0 & 0 & 2 & 1 \\
\hline Other & 1 & 3 & 7 & 4 \\
\hline \multicolumn{5}{|l|}{ Total receiving } \\
\hline SAARDs & 23 & 36 & 40 & 66 \\
\hline Significance & \multicolumn{2}{|c|}{ NS } & \multicolumn{2}{|c|}{$\mathrm{p}<0.001$} \\
\hline
\end{tabular}

China at the time of the study, whereas $59 \%$ of $\frac{\square}{\omega}$. Australian patients in the series had had gold? injections in the past. There was no statistical $\stackrel{\vec{S}}{\mathcal{S}}$ difference between current patients treated witho SAARDs as a group, but two thirds of the Australian patients had received SAARDs in the past $\frac{\bar{\sigma}}{\sigma}$ compared with $40 \%$ of the Chinese $(p<0 \cdot 001)$. Only $\mathbb{Q}$ one Chinese patient was taking a purely analgesic preparation compared with $41 \%$ of Australians. Corticosteroids were currently prescribed in $26 \%$. of Chinese patients and $39 \%$ of Australians. Tra- $\overrightarrow{\vec{\omega}}$ ditional Chinese therapy was being used by $13 \%$ of $\stackrel{\omega}{\omega}$ the Chinese patients but was not recorded in Australia, while folk or herbal remedies were recorded in $22 \%$ of Chinese and $5 \%$ of Australian patients. Despite the infrequency of peptic ulcers in $v$ ir Chinese $25 \%$ of this group were taking antacids and $N$ $6 \%$ 'antiulcerants'. The equivalent Australiano figures were $20 \%$ and $15 \%$. More Australians were taking sedatives, antidepressants, and diuretics. $\subset$ Surgery for joints, tendons, and the carpal tunnel⿳亠口 syndrome had been undertaken in $40 \%$ of Austra- $\vec{\theta}$ lian and only $2 \%$ of Chinese patients.

$X$ RAY RESULTS

$x$ Ray results are summarised in Table 8 . Striking differences were noted in both the assessments of hands and feet, with higher grades representing more severe disease being shown in the Australian $\mathbb{Q}$ series $(p<0 \cdot 001)$. Differences in the chest $x$ rays $\Rightarrow$ were not as marked, with slightly less than half of the Chinese series and slightly less than two thirds of the Australian series having normal chest $x$ rays. Old chronic tuberculosis was more common in the Chinese series $(p<0.025)$.

LA BORATORY IN VESTIGATIONS

The latex test for the rheumatoid factor was positive in $70 \%$ of the Chinese series and $65 \%$ of the Australians, while antinuclear antibody positivity ino a titre of 1 in 40 or more occurred in $30 \%$ of Chinese

Table 8 x Ray results (in percentages)

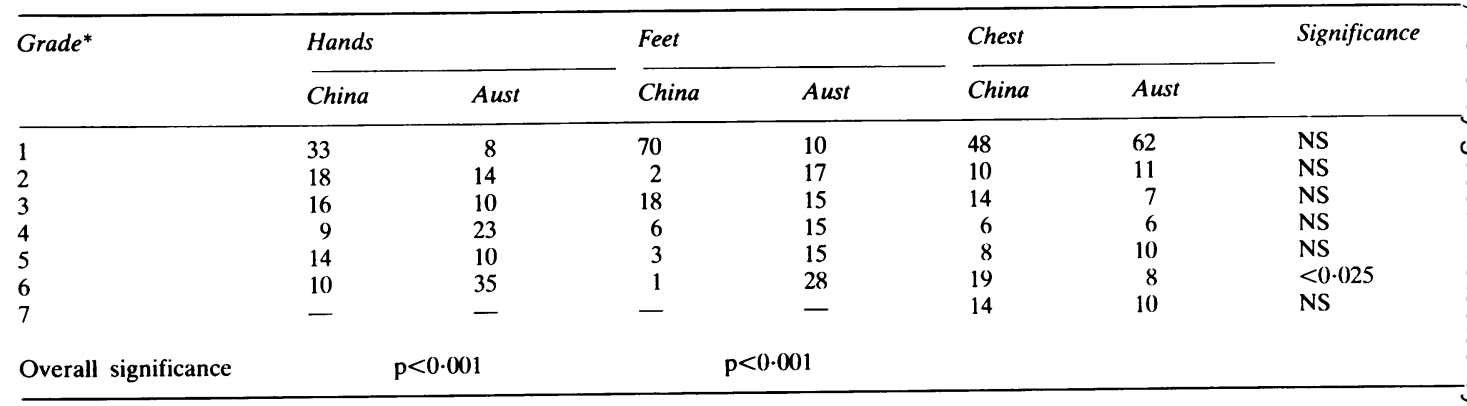

${ }^{*}$ For meaning of grades see text. 
Table 9 Haematology (mean values)

\begin{tabular}{lllll}
\hline & $\begin{array}{l}\mathrm{Hb} \\
(\mathrm{g} / \mathrm{dl})^{*}\end{array}$ & $\begin{array}{l}\text { White cells } \\
\times 10^{-9} / l\end{array}$ & $\begin{array}{l}\text { Platelets } \\
\times 10^{-9} / l\end{array}$ & $\begin{array}{l}\text { ESR } \\
(\mathrm{mm} / \mathrm{lst} h)\end{array}$ \\
\hline China & 10.6 & 6.0 & 148 & 50 \\
Australia & 13.0 & 8.0 & 390 & 34 \\
Significance & $\mathrm{p}<0.001$ & $\mathrm{p}<0.001$ & $\mathrm{p}<0.001$ & $\mathrm{p}<0.001$ \\
\hline
\end{tabular}

*SI conversion: $\mathrm{g} / \mathrm{dl} \times 10=\mathrm{g} / \mathrm{l}$.

and $24 \%$ of Australians. Neither of these differences reached significance. The results of haemoglobin, white cell count, platelet count, and ESR are shown in Table 9. Impairment in renal function as measured by serum creatinine and proteinuria was uncommon in both series.

\section{Discussion}

The aim of the study was not to perform a survey of RA as it affects a sample of the total population in Australia and China but rather to consider the pattern of disease as it presents to a rheumatology unit in the two countries. The patients therefore would be more likely to have established and more severe disease than would be seen by a doctor of first contact. Even when this disadvantage is accepted the results recorded here shed some light on the hypothesis that the severity of RA in China is less than in western countries. ${ }^{6}$ Severity measured by joint deformities, as particularly shown by ulnar deviation of the fingers and flexion contractures of the elbows, was significantly greater in the Australian series. Severity was even more dramatically shown in the differences in the $x$ rays of the hands and feet. With regard to extra-articular manifestations the suggestion ${ }^{6}$ that subcutaneous and tendon rheumatoid nodules are uncommon in RA patients in China has been confirmed. Raynaud's phenomenon was much more common in the Australian series, and of pointers to vasculitis, only an increase in leg ulcers in the Australian series might suggest that there was a difference. Eye complications were rare in both groups apart from the positive Schirmer's test for lacrymal secretion, where impairment was more common in the Chinese. Pulmonary, cardiac, and neurological signs were also uncommon in both series and chest $x$ rays showed no major differences apart from the increase in old tuberculosis noted in the Chinese series. Lymphadenopathy and hepatomegaly but not splenomegaly were commoner in the Chinese, and it is possible that the increased axillary and inguinal lymphadenopathy at least could be related to the more active joint disease demonstrated in the Chinese.
Further consideration of the examination of the joints points to another obvious difference in that joint tenderness was more marked in the Chinese and this involved PIP, MCP, wrists, elbows, shoulders, and MTP joints. Swelling, however, was more common only in the PIP joints. The significantly higher ESR results fit in with the general conclusion that more acute inflammation in joints occurs in the Chinese series as opposed to more chronic changes among Australian patients. The significantly lower haemoglobin level in the Chinese could also indicate increased disease activity, though there are a number of other possible explanations. The reason for the low platelet count in the Chinese has yet to be explained.

From the point of view of comparability there were no significant differences in seropositivity for the rheumatoid factor nor for the frequency of ANA. Disease onset was the same, and more surprisingly in view of the greater severity of disease in the Australian series there were no significant differences in the disease course.

The explanation for the increased frequency of peptic ulcer in Australia does not appear to be related to major differences in the usage of drugs. The majority of both series were taking NSAIDs even though these varied in type between the two countries. Despite the fact that more salicylates were used in Australia this did not correlate with peptic ulcer occurrence. Chinese doctors tend to use antacids routinely with patients taking NSAIDs and this may be the reason why so many Chinese patients were taking antacid preparations (25\%). The more widespread use of SAARDs could possibly reflect the more severe and chronic disease as seen in the Australian series. The availability of specific SAARDs clearly varied, with gold being the most popular drug in Australia and penicillamine in China. Only small numbers of patients in these series were on immunosuppressive drugs.

The frequent use of surgery in Australia possibly relates to the more severe and chronic changes but also must in part be due to a difference in availability of procedures such as total hip and total knee replacements in the two countries.

When the differences that have been shown between the two series are considered the major influence on the disease picture of the longer disease duration and the higher mean age of patients in the Australian series must be acknowledged. Certainly some of the more chronic changes as evidenced by deformity, decreased range of joint motion, and $x$ ray changes are at least partly related to these factors. The increased frequency of ischaemic heart disease in Australians could also reflect an age factor. Despite these limitations it should be noted 
that the mean disease duration in China was 6.3 years and one can therefore anticipate that the pattern of disease with regard to severity and extra-articular manifestations including nodules would be established. Little evidence has been provided that other visceral manifestations and in particular vasculitis are more common problems in RA in Australia than in China. With regard to treatment, apart from the infrequent use of joint surgery in China, similarities of approach outnumber differences. Disease chronicity and availability of drugs explain most of the differences that were recorded.

We thank Dr John Mathews for essential advice and support and Peter Derrick and Chris Muirden for their help with the statistical analysis. We also acknowledge support from the Greek Fund for $\frac{\square}{\oplus}$. Arthritis Research of The Royal Melbourne Hospital.

\section{References}

1 Shichikawa K, Takenaka Y, Maeda A, et al. A longitudinal population survey of RA in a rural district in Wakayama. Ryumachi 1981; 21 (suppl): 35-43.

2 Lawrence J S. Prevalence of rheumatoid arthritis. Ann Rheum Dis 1961; 20: 11-7.

3 Mikkelsen W M, Dodge H J, Duff I F, et al. Estimates of the prevalence of rheumatic diseases in the population of Tecumseh $\overrightarrow{0}$ Michigan 1959-60. J Chronic Dis 1967; 20: 351-69.

4 Rose B S. Prior I A M. A survey of rheumatism in a rural New $\vec{C}$ Zealand Maori community. Ann Rheum Dis 1963; 22: 410-5.

5 Beasley R P, Bennett P H. Chien C L. Low prevalence of rheumatoid arthritis in Chinese: prevalence survey in a rural community. J Rheumatol 1983; 10 (suppl): 11-5.

6 Chang N C. Rheumatic diseases in China. J Rheumatol 1983; 10 जे (suppl): 41-5. 